\title{
EXTREME RESIDUES OF DEDEKIND ZETA FUNCTIONS
}

\author{
PETER J. CHO AND HENRY H. KIM*
}

\begin{abstract}
In a family of $S_{d+1}$-fields $(d=2,3,4)$, we obtain the true upper and lower bound of the residues of Dedekind zeta functions except for a density zero set. For $S_{5}$-fields, we need to assume the strong Artin conjecture. We also show that there exists an infinite family of number fields with the upper and lower bound, resp.
\end{abstract}

\section{INTRODUCTION}

For a quadratic extension $K=\mathbb{Q}(\sqrt{D})$ with a fundamental discriminant $D, \operatorname{Res}_{s=1} \zeta_{K}(s)=$ $L\left(1, \chi_{D}\right)$, where $\chi_{D}=\left(\frac{D}{\cdot}\right)$ is the quadratic character. In this case, Littlewood [10] obtained the bound

$$
\left(\frac{1}{2}+o(1)\right) \frac{\zeta(2)}{e^{\gamma} \log \log |D|} \leq L\left(1, \chi_{D}\right) \leq(2+o(1)) e^{\gamma} \log \log |D|
$$

under GRH, where $\gamma$ is the Euler-Mascheroni constant. Under the same hypothesis, he also constructed an infinite family of quadratic fields with $L\left(1, \chi_{D}\right) \geq(1+o(1)) e^{\gamma} \log \log |D|$ and an infinite family of quadratic fields with $L\left(1, \chi_{D}\right) \leq(1+o(1)) \frac{\zeta(2)}{e^{\gamma} \log \log |D|}$. Later, Chowla [3] established the latter omega result unconditionally. It has been conjectured that the true upper and lower bounds are $(1+o(1)) e^{\gamma} \log \log |D|$ and $(1+o(1)) \frac{\zeta(2)}{e^{\gamma} \log \log |D|}$, resp. In [11], Montgomery and Vaughan considered the distribution of $L\left(1, \chi_{D}\right)$ via random variables which take \pm 1 with equal probability. They proposed three conjectures which support the expected bounds. In [5], some of the conjectures were proved by Granville and Soundararajan.

For a number field $K$ of degree $d+1$, the lower bound and the upper bound of $\operatorname{Res}_{s=1} \zeta_{K}(s)$ under GRH and the strong Artin conjecture for $\zeta_{K}(s) / \zeta(s)$ are

$$
\left(\frac{1}{2}+o(1)\right) \frac{\zeta(d+1)}{e^{\gamma} \log \log \left|D_{K}\right|} \leq \operatorname{Res}_{s=1} \zeta_{K}(s) \leq(2+o(1))^{d}\left(e^{\gamma} \log \log \left|D_{K}\right|\right)^{d},
$$

2010 Mathematics Subject Classification. Primary 11R42, Secondary 11M41.

Key words and phrases. Dedekind zeta functions; Artin $L$-functions; extreme values.

* partially supported by an NSERC grant. 
where $D_{K}$ is the discriminant of a number field $K$. The proof of (1.1) is given in Section 3 since at least the upper bound is well-known but it is hard to find its proof in the literature.

As in the quadratic extension case, we may conjecture that $(1+o(1))\left(e^{\gamma} \log \log \left|D_{K}\right|\right)^{d}$ and $(1+o(1)) \frac{\zeta(d+1)}{e^{\gamma} \log \log \left|D_{K}\right|}$ are the true upper and lower bound, resp. In this paper, we show that it is the case except for a density zero set in a family of number fields. A number field $K$ of degree $d+1$ is called a $S_{d+1}$-field if its Galois closure over $\mathbb{Q}$ is an $S_{d+1}$ Galois extension. For a $S_{d+1}$-field $K$, we have a decomposition of $\zeta_{K}(s)$ :

$$
\zeta_{K}(s)=\zeta(s) L(s, \rho, \widehat{K} / Q),
$$

where $\widehat{K}$ is the Galois closure of $K$ over $\mathbb{Q}$ and $\rho$ is the standard representation of $S_{d+1}$. For simplicity, we denote $L(s, \rho, \widehat{K} / Q)$ by $L(s, \rho)$. Hence $\operatorname{Res}_{s=1} \zeta_{K}(s)=L(1, \rho)$. Then, our first main theorem is

Theorem 1.2. Let $L(X)$ be a set of $S_{d+1}$-fields with $X / 2 \leq\left|D_{K}\right| \leq X, d+1=3,4$ and 5 . For $S_{5}$ fields, we assume the strong Artin conjecture for $L(s, \rho)$. Then, except for $O\left(X e^{-c^{\prime} \frac{\log X}{\log \log X} \log \log \log X}\right)$ $L$-functions for some constant $c^{\prime}>0$,

$$
(1+o(1)) \frac{\zeta(d+1)}{e^{\gamma} \log \log \left|D_{K}\right|} \leq L(1, \rho) \leq(1+o(1))\left(e^{\gamma} \log \log \left|D_{K}\right|\right)^{d} .
$$

where $o(1)=O\left(\frac{1}{\left(\log \log \left|D_{K}\right|\right)^{1 / 2}}\right)$.

Furthermore, under the same hypothesis, we construct an infinite family of $S_{d+1}$-fields with extreme residue values.

Theorem 1.3. Let $d+1=3,4$, and 5. For $d+1=5$, we assume the strong Artin conjecture. Then

(1) The number of $S_{d+1}$-fields $K$ of signature $\left(r_{1}, r_{2}\right)$ with $\frac{X}{2} \leq\left|D_{K}\right| \leq X$ for which

$$
\begin{aligned}
L(1, \rho) & =\prod_{p \leq y}\left(1-p^{-1}\right)^{-d}\left(1+O\left(\frac{1}{\left(\log \log \left|D_{K}\right|\right)^{1 / 2}}\right)\right) \\
& =\left(e^{\gamma} \log \log \left|D_{K}\right|\right)^{d}\left(1+O\left(\frac{1}{\left(\log \log \left|D_{K}\right|\right)^{1 / 2}}\right)\right)
\end{aligned}
$$

is $\geq A\left(r_{2}\right) X \exp \left(-\log \left|S_{d+1}\right| \cdot \frac{\log X}{\log \log X}-\log \log \log X\right)$.

(2) The number of $S_{d+1}$-fields $K$ of signature $\left(r_{1}, r_{2}\right)$ with $\frac{X}{2} \leq\left|D_{K}\right| \leq X$ for which

$$
L(1, \rho)=\frac{\zeta(d+1)}{e^{\gamma} \log \log \left|D_{K}\right|}\left(1+O\left(\frac{1}{(\log \log X)^{1 / 2}}\right)\right)
$$




$$
i s \geq A\left(r_{2}\right) X \exp \left(-\log \frac{\left|S_{d+1}\right|}{(d+1)} \cdot \frac{\log X}{\log \log X}-\log \log \log X\right) \text {. }
$$

We also construct an infinite family of $S_{d+1}$-fields with bounded residues.

Theorem 1.4. Let $d+1=3,4$, and 5. For $d+1=5$, we assume the strong Artin conjecture.

Then the number of $S_{d+1}$-fields $K$ of signature $\left(r_{1}, r_{2}\right)$ with $\frac{X}{2} \leq\left|D_{K}\right| \leq X$ for which

$$
L(1, \rho)=\left\{\begin{array}{ll}
\zeta(2)^{\frac{d}{2}}(1+o(1)), & \text { if } d \text { is even } \\
\zeta(2)^{\frac{d-3}{2}} \zeta(3)(1+o(1)), & \text { if } d \geq 3 \text { is odd. }
\end{array} .\right.
$$

is $\geq A\left(r_{2}\right) X \exp \left(-\log \frac{\left|S_{d+1}\right|}{|C|} \cdot \frac{\log X}{\log \log X}-\log \log \log X\right)$, where

$$
C=\left\{\begin{array}{ll}
(1,2)(3,4) \cdots(d-1, d), & \text { if } d \text { is even } \\
(1,2)(3,4) \cdots(d-4, d-3)(d-2, d-1, d), & \text { if } d \text { is odd }
\end{array} .\right.
$$

This work is motivated by the work of Lamzouri [8, 9], who constructed primitive characters $\chi$ with large values of $L(1, \chi)$. Basically, we follow [8, 9, 5, 11]. The arguments in [8] are easily extended. However, obtaining an analogue of Proposition 2.4 in [8] is a main obstacle to extend his method. It is resolved in Proposition 4.3.

\section{Counting number fields With LOCAl COnditions}

Let $K$ be a $S_{d+1}$-field of signature $\left(r_{1}, r_{2}\right)$ for $d+1 \geq 3$. We assume that we can count $S_{d+1}$-fields with finitely many local conditions. Namely, let $\mathcal{S}=\left(\mathcal{L C}_{p}\right)$ be a finite set of local conditions: $\mathcal{L C}_{p}=\mathcal{S}_{p, C}$ means that $p$ is unramified and the conjugacy class of Frob ${ }_{p}$ is $C$. Define $\left|\mathcal{S}_{p, C}\right|=\frac{|C|}{\left|S_{n}\right|(1+f(p))}$ for some function $f(p)$ which satisfies $f(p)=O\left(\frac{1}{p}\right)$. There are also several splitting types of ramified primes, which are denoted by $r_{1}, r_{2}, \ldots, r_{w}: \mathcal{L C}_{p}=\mathcal{S}_{p, r_{j}}$ means that $p$ is ramified and its splitting type is $r_{j}$. We assume that there are positive valued functions $c_{1}(p)$, $c_{2}(p), \ldots, c_{w}(p)$ with $\sum_{i=1}^{w} c_{i}(p)=f(p)$ and define $\left|\mathcal{S}_{p, r_{i}}\right|=\frac{c_{i}(p)}{1+f(p)}$. We define the local condition $\mathcal{L C}_{p}=S_{p, r}$ which means that $p$ is ramified, i.e, $r=r_{j}$ for some $j$. Define $\left|\mathcal{S}_{p, r}\right|=\frac{f(p)}{1+f p}$. Let $|\mathcal{S}|=\prod_{p}\left|\mathcal{L C}_{p}\right|$.

Let $L(X)^{r_{2}}$ be the set of $S_{d+1}$-fields $K$ of signature $\left(r_{1}, r_{2}\right)$ with $\frac{X}{2}<\left|D_{K}\right|<X$, and let $L(X ; \mathcal{S})^{r_{2}}$ be the set of $S_{d+1}$-fields $K$ of signature $\left(r_{1}, r_{2}\right)$ with $\frac{X}{2}<\left|D_{K}\right|<X$ and the local conditions $\mathcal{S}$. Then we have 
Conjecture 2.1. For some positive constants $\delta<1$ and $\kappa$,

$$
\begin{aligned}
\left|L(X)^{r_{2}}\right| & =A\left(r_{2}\right) X+O\left(X^{\delta}\right), \\
\left|L(X ; \mathcal{S})^{r_{2}}\right| & =|\mathcal{S}| A\left(r_{2}\right) X+O\left(\left(\prod_{p \in S} p\right)^{\kappa} X^{\delta}\right),
\end{aligned}
$$

where the implied constant is uniformly bounded for $p$ and local conditions at $p$.

It is worth noting here that we can control only all the primes up to $c \log X$, where $c<(1-\delta) / \kappa$. If we impose local conditions for all $p \leq c^{\prime} \log X$ with $c^{\prime} \geq(1-\delta) / \kappa$, the error term in Conjecture 2.1 would be larger than the size of $L(X)^{r_{2}}$.

For $S_{3}$-fields, the conjecture was shown by Taniguchi and Thorne [12]. In [2]1, we proved that Conjecture 2.1 is true for $S_{4}$ and $S_{5}$-fields.

\section{Formula FOR $L(1, \rho)$ UNDER a CERTAin ZERO-FREE REGION}

In this paper, we assume the strong Artin conjecture, namely, the Artin $L$-function $L(s, \rho)$ is an automorphic representation of $G L_{d}$. This is true for $S_{3}$-fields and $S_{4}$-fields. It implies the Artin conjecture, namely, $L(s, \rho)$ is entire. For this section, we only need the Artin conjecture. However, in Section 4, we need the strong Artin conjecture in order to use Kowalski-Michel zero density theorem [7]. We find an expression of $L(1, \rho)$ as a product over small primes under assumption that $L(s, \rho)$ has a certain zero-free region. Here all the implicit constants only depend on the degree $d$ of $L(s, \rho)$.

For $\operatorname{Re}(s)>1, L(s, \rho)$ has the Euler product:

$$
L(s, \rho)=\prod_{p} \prod_{i=1}^{d}\left(1-\frac{\alpha_{i}(p)}{p^{s}}\right)^{-1} .
$$

Then, for $\operatorname{Re}(s)>1$,

$$
\log L(s, \rho)=\sum_{n=2}^{\infty} \frac{\Lambda(n) a_{\rho}(n)}{n^{s} \log n},
$$

where $a_{\rho}\left(p^{k}\right)=\alpha_{1}(p)^{k}+\cdots+\alpha_{d}(p)^{k}$. First, we show that when $L(s, \rho)$ has a certain zero-free region, the value $\log L(1, \rho)$ is determined by a short sum.

\footnotetext{
${ }^{1}$ In [2], we used the Greek letter $\gamma$ in place of $\kappa$. However, $\gamma$ is taken for the Euler-Mascheroni constant in this article.
} 
Proposition 3.1. If $L(s, \rho)$ is entire and is zero-free in the rectangle $[\alpha, 1] \times[-x, x]$, where $x=(\log N)^{\beta}, \beta(1-\alpha)>2$, and $N$ is the conductor of $\rho$, then

$$
\log L(1, \rho)=\sum_{n<x} \frac{\Lambda(n) a_{\rho}(n)}{n \log n}+O\left((\log N)^{-1}\right) .
$$

Proof. By Perron's formula,

$$
\frac{1}{2 \pi i} \int_{c-i x}^{c+i x} \log L(1+s, \rho) \frac{x^{s}}{s} d s=\sum_{n<x} \frac{\Lambda(n) a_{\rho}(n)}{n \log n}+O\left(\frac{\log x}{x}\right) .
$$

where $c=\frac{1}{\log x}$.

Now move the contour to $\operatorname{Re}(s)=\alpha-1+\frac{1}{\log x}$. We get the residue $\log L(1, \rho)$ at $s=0$. So the left hand side is $\log L(1, \rho)$ plus

$$
\frac{1}{2 \pi i}\left(\int_{c-i x}^{\alpha-1+c-i x}+\int_{\alpha-1+c-i x}^{\alpha-1+c+i x}+\int_{\alpha-1+c+i x}^{c+i x}\right) \log L(1+s, \rho) \frac{x^{s}}{s} d s .
$$

In order to estimate $|\log L(s, \rho)|$ for $\alpha+c \leq R e(s) \leq 1+c$, we follow [6, Lemma 8.1]: Consider the circles with centre $2+i t$ and radii $r=2-\sigma<R=2-\alpha$. By the assumption, $\log L(s, \rho)$ is holomorphic inside the larger circle. By Daileda [4, page 222], for $\frac{1}{2}<R e(s) \leq \frac{3}{2},|L(s, \rho)| \leq$ $N^{\frac{1}{2}}(|s|+1)^{\frac{d}{2}}$. Hence Re $\log L(s, \rho)=\log |\log L(s, \rho)| \ll \log N+\log (|s|+1)$. Clearly, if $\operatorname{Re}(s) \geq \frac{3}{2}$, $|\log L(s, \rho)|=O(1)$. By the Borel-Carathéodory theorem,

$|\log L(s, \rho)| \leq \frac{2 r}{R-r} \max _{z-(2+i t) \mid=R} R e \log L(z, \rho)+\frac{R+r}{R-r}|\log L(2+i t, \rho)| \ll(\log x)(\log N+\log (|s|+1))$.

Hence the integral is majorized by $x^{\alpha-1}(\log N)(\log x)^{2}$. Since $\beta(1-\alpha)>2, x^{\alpha-1}(\log N)(\log x)^{2} \ll$ $(\log N)^{-1}$.

Remark 3.3. Assume that $L(s, \rho)$ satisfies GRH. Take $\alpha=1 / 2+\epsilon^{2}$ and $\beta=2+\epsilon$. Then, from the above proof, we can see that

$$
\log L(1, \rho)=\sum_{n<(\log N)^{2+\epsilon}} \frac{\Lambda(n) a_{\rho}(n)}{n \log n}+O\left(\frac{\log \log N}{(\log N)^{\frac{\epsilon}{2}-\left(2 \epsilon^{2}+\epsilon^{3}\right)}}\right),
$$

for any $\epsilon>0$.

Now, using Proposition 3.1, we express $L(1, \rho)$ as a product over small primes. We omit $p$ from $\alpha_{i}(p)$ for simplicity.

$$
\sum_{n<x} \frac{\Lambda(n) a_{\rho}(n)}{n \log n}=\sum_{k, p^{k}<x} \frac{\alpha_{1}^{k}+\cdots+\alpha_{d}^{k}}{k p^{k}}=\sum_{p<x} \sum_{i=1}^{d} \sum_{k<\frac{\log x}{\log p}} \frac{1}{k}\left(\alpha_{i} p^{-1}\right)^{k} .
$$


Here

where

$$
\sum_{k<\frac{\log x}{\log p}} \frac{1}{k}\left(\alpha_{i} p^{-1}\right)^{k}=-\log \left(1-\alpha_{i} p^{-1}\right)+A_{p}
$$

$$
\left|A_{p}\right| \leq \sum_{k \geq \frac{\log x}{\log p}} \frac{1}{k} p^{-k} \leq \frac{\log p}{\log x} \cdot \frac{p^{-\frac{\log x}{\log p}}}{1-p^{-1}}
$$

Here $p^{\frac{\log x}{\log p}}=x$. Hence

$$
(\underline{3.4})=-\sum_{p<x} \sum_{i=1}^{d} \log \left(1-\alpha_{i} p^{-1}\right)+d \sum_{p<x} A_{p} \text {. }
$$

Here

$$
\sum_{p<x}\left|A_{p}\right| \leq \frac{1}{x \log x} \sum_{p<x} \frac{\log p}{1-p^{-1}} \leq \frac{2}{\log x}
$$

Therefore, it is summarized as follows:

Proposition 3.5. If $L(s, \rho)$ is entire and is zero-free in the rectangle $[\alpha, 1] \times[-x, x]$, where $x=(\log N)^{\beta}, \beta(1-\alpha)>2$, and $N$ is the conductor of $\rho$, then

$$
L(1, \rho)=\prod_{p<x} \prod_{i=1}^{d}\left(1-\alpha_{i} p^{-1}\right)^{-1}\left(1+O\left(\frac{1}{\log x}\right)\right) .
$$

Furthermore, if $L(s, \rho)$ satisfies $G R H$, then

$$
L(1, \rho)=\prod_{p<(\log N)^{2+\epsilon}} \prod_{i=1}^{d}\left(1-\alpha_{i} p^{-1}\right)^{-1}\left(1+O\left(\frac{1}{\log \log N}\right)\right) .
$$

In order to find the upper and lower bound of $L(1, \rho)$, we examine the Euler product: Let $C$ be a conjugacy class of $S_{d+1}$, and let $C$ be a product of $d_{1}, \cdots, d_{k}$ cycles, where $d_{i} \geq 1$ for all $i$ and $d_{1}+\cdots+d_{k}=d+1$. Then if $\operatorname{Frob}_{p} \in C,(1-X) \prod_{i=1}^{d}\left(1-\alpha_{i} X\right)=\left(1-X^{d_{1}}\right) \cdots\left(1-X^{d_{k}}\right)$. Hence

$$
\prod_{i=1}^{d}\left(1-\alpha_{i} p^{-1}\right)^{-1}=\left(1-p^{-1}\right)\left(1-p^{-d_{1}}\right)^{-1} \cdots\left(1-p^{-d_{k}}\right)^{-1} .
$$

Now we use Mertens' theorem:

$$
\prod_{p \leq y}\left(1-p^{-1}\right)^{-1}=e^{\gamma}(1+o(1)) \log y .
$$

Also $\prod_{p \leq y}\left(1-p^{-n}\right)^{-1}=\zeta(n)\left(1+O\left(\frac{1}{y \log y}\right)\right)$ if $n \geq 2$. 
Hence the upper bound of $\prod_{i=1}^{d}\left(1-\alpha_{i} p^{-1}\right)^{-1}$ is when $C=1$, and it is $\left(1-p^{-1}\right)^{-d}$. The lower bound is when $C=(1, \cdots, d+1)$, and it is $\left(1-p^{-1}\right)\left(1-p^{-d-1}\right)^{-1}$. Moreover, it takes only the values $\left(1-p^{-e_{1}}\right)^{-a_{1}} \cdots\left(1-p^{-e_{l}}\right)^{-a_{l}}\left(1-p^{-1}\right)^{a_{0}}$, where $e_{1}, \ldots, e_{l} \geq 2$, and $-d \leq a_{0} \leq 1$. Here $a_{0}=1$ only when $a_{1} e_{1}+\cdots+a_{l} e_{l}=d+1$. We summarize it as

$$
\left(1-p^{-1}\right)\left(1-p^{-d-1}\right)^{-1} \leq \prod_{i=1}^{d}\left(1-\alpha_{i} p^{-1}\right)^{-1} \leq\left(1-p^{-1}\right)^{-d} .
$$

We note that (3.7) is true even if $p$ is ramified, i.e., when some of $\alpha_{i}$ 's are zero. Hence by the above proposition, under GRH and the strong Artin conjecture for $L(s, \rho)$, for any $\epsilon>0$,

$$
\frac{\zeta(d+1)}{(2+\epsilon) e^{\gamma} \log \log N}(1+o(1)) \leq L(1, \rho) \leq\left(e^{\gamma}(2+\epsilon) \log \log N\right)^{d}(1+o(1)) .
$$

Since $\epsilon$ is arbitrarily small, we showed

$$
\left(\frac{1}{2}+o(1)\right) \frac{\zeta(d+1)}{e^{\gamma} \log \log N} \leq L(1, \rho) \leq(2+o(1))^{d}\left(e^{\gamma} \log \log N\right)^{d} .
$$

\section{Extreme Residue values}

4.1. True upper and lower bound. For simplicity, we denote $L(X)^{r_{2}}$ by $L(X)$. Let $y=$ $c_{1} \log X$ with $c_{1}>0$. Recall that in Proposition 3.1, the conductor of $L(s, \rho)$ is $\left|D_{K}\right|$, and $\frac{X}{2}<\left|D_{K}\right|<X$, and $x=(\log X)^{\beta}$ for some $\beta$.

In this section we show that except for $O\left(X e^{-c^{\prime} \frac{\log X}{\log \log X} \log \log \log X}\right)$ in $L(X)$, the lower bound and upper bound on $L(1, \rho)$ are

$$
(1+o(1)) \frac{\zeta(d+1)}{e^{\gamma}\left(\log \log \left|D_{K}\right|\right)}, \quad(1+o(1))\left(e^{\gamma} \log \log \left|D_{K}\right|\right)^{d}, \quad \text { resp. }
$$

We apply Kowalski-Michel zero density theorem [7] to the family $L(X)$. Then except for $O\left((\log X)^{\beta B} X^{\left(\frac{5 d}{2}+1\right) \frac{1-\alpha}{2 \alpha-1}}\right) L$-functions, every $L$-function $L(s, \rho)$ in $L(X)$ is zero-free on $[\alpha, 1] \times$ $\left[-(\log X)^{\beta},(\log X)^{\beta}\right]$ with $\beta(1-\alpha)>2$. Here $B$ is a constant depending on the family $L(X)$. We refer to [1] for the detail.

Since except for $O\left((\log X)^{\beta B} X^{\left(\frac{5 d}{2}+1\right) \frac{1-\alpha}{2 \alpha-1}}\right) L$-functions, the $L$-functions in $L(X)$ have the desired zero-free region, we apply Proposition 3.5 to the $L$-functions in $L(X)$ to obtain

$$
L(1, \rho)=\prod_{p<x} \prod_{i=1}^{d}\left(1-\alpha_{i} p^{-1}\right)^{-1}\left(1+O\left(\frac{1}{\log x}\right)\right) .
$$


Since

$$
\sum_{y<p<x} \frac{1}{p^{2}} \leq \sum_{p>y} \frac{1}{p^{2}} \leq \frac{2}{y \log y}
$$

we can show

$$
\prod_{y<p<x} \prod_{i=1}^{d}\left(1-\alpha_{i} p^{-1}\right)^{-1}=\exp \left(\sum_{y<p<x} \frac{a_{\rho}(p)}{p}\right)\left(1+O\left(\frac{1}{y \log y}\right)\right) .
$$

We prove

Proposition 4.1. Except for $O\left(X e^{-c^{\prime} \frac{\log X}{\log \log X} \log \log \log X}\right)$ L-functions in $L(X)$ for some constant $c^{\prime}>0$, L-functions in $L(X)$ satisfy

$$
\left|\sum_{y<p<x} \frac{a_{\rho}(p)}{p}\right| \leq \frac{1}{(\log \log X)^{1 / 2}} .
$$

Hence, for $L$-functions which have the desired zero-free region and satisfy (4.2),

$$
L(1, \rho)=\prod_{p \leq y} \prod_{i=1}^{d}\left(1-\alpha_{i} p^{-1}\right)^{-1}\left(1+\frac{1}{\left(\log \log \left|D_{K}\right|\right)^{1 / 2}}\right) .
$$

This and (3.7) implies immediately Theorem 1.2 .

In order to prove Proposition 4.1, we follow the idea in [8]. Namely we prove

Proposition 4.3. Let $y=c_{1} \log X$ and $r \leq c_{2} \frac{\log X}{\log \log X}$ for some positive constants $c_{1}$ and $c_{2}$. Then,

$$
\sum_{\rho \in L(X)}\left(\sum_{y<p<x} \frac{a_{\rho}(p)}{p}\right)^{2 r} \ll 2^{2 r-1} d^{2 r} \frac{(2 r) !}{r !} \frac{2^{2 r}}{(y \log y)^{r}} X
$$

with an absolute implied constant.

By Stirling's formula, $2^{2 r-1} d^{2 r} \frac{(2 r) !}{r !} \frac{2^{2 r}}{(y \log y)^{r}} \ll\left(\frac{c d^{2} r}{y \log y}\right)^{r}$ for a constant $c$.

Proof. By multinomial formula, the left hand side is

$$
\sum_{\rho \in L(X)} \sum_{u=1}^{2 r} \frac{1}{u !} \sum_{r_{1}, \ldots, r_{u}}^{(1)} \frac{(2 r) !}{r_{1} ! \cdots r_{u} !} \sum_{p_{1}, \ldots, p_{u}}^{(2)} \frac{a_{\rho}\left(p_{1}\right)^{r_{1}} \cdots a_{\rho}\left(p_{u}\right)^{r_{u}}}{p_{1}^{r_{1}} \cdots p_{u}^{r_{u}}}
$$

where $\sum_{r_{1}, \ldots, r_{u}}^{(1)}$ means the sum over the $u$-tuples $\left(r_{1}, \ldots, r_{u}\right)$ of positive integers such that $r_{1}+$ $\cdots+r_{u}=2 r$, and $\sum_{p_{1}, \ldots, p_{u}}^{(2)}$ means the sum over the $u$-tuples $\left(p_{1}, \ldots, p_{u}\right)$ of distinct primes such 
that $y<p_{i}<x$ for each $i$. Write

$$
\text { (4.4) }=\sum_{u=1}^{2 r} \sum_{r_{1}, \ldots, r_{u}}^{(1)} \frac{(2 r) !}{r_{1} ! \cdots r_{u} !} \frac{1}{u !} \sum_{p_{1}, \ldots, p_{u}}^{(2)} \frac{1}{p_{1}^{r_{1}} \cdots p_{u}^{r_{u}}}\left(\sum_{\rho \in L(X)} a_{\rho}\left(p_{1}\right)^{r_{1}} \cdots a_{\rho}\left(p_{u}\right)^{r_{u}}\right) \text {. }
$$

We will show that for any composition $r_{1}+r_{2}+\cdots+r_{u}=2 r$,

$$
\frac{(2 r) !}{r_{1} ! \cdots r_{u} !} \frac{1}{u !} \sum_{p_{1}, \ldots, p_{u}}^{(2)} \frac{1}{p_{1}^{r_{1}} \cdots p_{u}^{r_{u}}}\left(\sum_{\rho \in L(X)} a_{\rho}\left(p_{1}\right)^{r_{1}} \cdots a_{\rho}\left(p_{u}\right)^{r_{u}}\right) \ll d^{2 r} X \frac{(2 r) !}{r !} \frac{2^{2 r}}{(y \log y)^{r}}
$$

Since the number of compositions of $2 r$ is $2^{2 r-1}$, it implies that

$$
\text { (4.4) } \ll 2^{2 r-1} d^{2 r} \frac{(2 r) !}{r !} \frac{2^{2 r}}{(y \log y)^{r}} X .
$$

First, we consider compositions with $r_{i} \geq 2$ for all $i$. Then by using the trivial bound,

$$
\begin{aligned}
& \sum_{p_{1}, \ldots, p_{u}}^{(2)} \frac{1}{p_{1}^{r_{1}} \cdots p_{u}^{r_{u}}}\left(\sum_{\rho \in L(X)} a_{\rho}\left(p_{1}\right)^{r_{1}} \cdots a_{\rho}\left(p_{u}\right)^{r_{u}}\right) \ll d^{2 r} X\left(\sum_{y<p_{1}<x} \frac{1}{p_{1}^{r_{1}}}\right) \cdots\left(\sum_{y<p_{u}<x} \frac{1}{p_{u}^{r_{u}}}\right) \\
& \ll d^{2 r} X \frac{2^{2 r}}{(y \log y)^{r}}\left(\frac{\log y}{y}\right)^{r-u} .
\end{aligned}
$$

Hence (4.5) is proved once we show that for any $r_{1}, \ldots, r_{u}$ such that $r_{1}+\cdots+r_{u}=2 r$, and $r_{i} \geq 2$ for all $i$,

$$
\frac{1}{u ! r_{1} ! \cdots r_{u} !}\left(\frac{\log y}{y}\right)^{r-u} \leq \frac{1}{r !}
$$

or equivalently

$$
\frac{r !}{u ! r_{1} ! \cdots r_{u} !} \leq\left(\frac{y}{\log y}\right)^{r-u}
$$

Since $r_{i} \geq 2$ for all $i=1,2, \ldots, u$, we have $u \leq r$. Since $y=c_{1} \log X$ and $r \leq c_{2} \frac{\log X}{\log \log X}$, $r \leq \frac{y}{\log y}$ for sufficiently small $c_{2}$. Then

$$
\frac{r !}{u ! r_{1} ! \cdots r_{u} !} \leq \frac{r !}{u !}=r(r-1) \cdots(r-u+1) \leq r^{r-u} \leq\left(\frac{y}{\log y}\right)^{r-u}
$$

Next, suppose $r_{i}=1$ for some $i$. We may assume that $r_{1}+\cdots+r_{m}+r_{m+1}+\cdots+r_{u}=2 r$, $r_{1}=\ldots=r_{m}=1$, and $r_{m+1}>1, \ldots, r_{u}>1$. First, we need a technical combinatorial lemma.

Lemma 4.7. Let $r_{i}$ 's be as above. Then

$$
\frac{1}{u !} \cdot \frac{1}{r_{1} ! r_{2} ! \ldots r_{m} ! r_{m+1} ! \ldots r_{u} !} \cdot \frac{y^{u}}{y^{m+r}} \cdot \frac{(\log y)^{r}}{(\log y)^{u}} \leq \frac{1}{r !} .
$$


Proof. First, we assume that $m$ is even. Then since $r_{m+1}, \ldots, r_{u} \geq 2$, and $r_{m+1}+\cdots+r_{u}=2 r-m$, by (4.6),

$$
\frac{\left(\frac{2 r-m}{2}\right) !}{(u-m) ! r_{m+1} ! \ldots r_{u} !} \leq\left(\frac{y}{\log y}\right)^{(r-m / 2)-(u-m)} \leq\left(\frac{y}{\log y}\right)^{r+m / 2-u}
$$

Hence

So

$$
\frac{1}{r_{m+1} ! \ldots r_{u} !} \leq \frac{(u-m) !}{(r-m / 2) !}\left(\frac{y}{\log y}\right)^{r+m / 2-u}
$$

$$
\begin{aligned}
\frac{1}{u !} \cdot \frac{1}{r_{1} ! r_{2} ! \ldots r_{m} ! r_{m+1} ! \ldots r_{u} !} \frac{y^{u}}{y^{m+r}} \frac{(\log y)^{r}}{(\log y)^{u}} & \leq \frac{(u-m) !}{u !} \frac{1}{(r-m / 2) !}\left(\frac{y}{\log y}\right)^{r+m / 2-u} \frac{y^{u}}{y^{m+r}} \frac{(\log y)^{r}}{(\log y)^{u}} \\
& \leq \frac{(u-m) !}{u !} \frac{1}{(r-m / 2) !} \frac{1}{(y \log y)^{m / 2}}
\end{aligned}
$$

Since $r<y$ and $\frac{(u-m) !}{u !}<1$,

$$
\frac{r !}{\left(r-\frac{m}{2}\right) !} \frac{(u-m) !}{u !} \leq(y \log y)^{m / 2}
$$

This implies

$$
\frac{(u-m) !}{u !} \frac{1}{(r-m / 2) !} \frac{1}{(y \log y)^{m / 2}} \leq \frac{1}{r !}
$$

Hence we have (4.8).

When $m$ is odd, we consider a composition of $2 r-m+3$ of the form:

$$
r_{m+1}^{\prime}=r_{m+1}, r_{m+2}^{\prime}=r_{m+2}, \ldots, r_{u}^{\prime}=r_{u}, \text { and } r_{u+1}^{\prime}=3 .
$$

With this composition, by (4.6),

$$
\frac{\left(\frac{2 r-m+3}{2}\right) !}{(u-m+1) ! r_{m+1} ! \ldots r_{u} ! 3 !}=\frac{\left(\frac{2 r-m+3}{2}\right) !}{(u-m+1) ! r_{m+1}^{\prime} ! \ldots r_{u}^{\prime} ! r_{u+1}^{\prime} !} \leq\left(\frac{y}{\log y}\right)^{r+m / 2+1 / 2-u}
$$

As we did for the case of even $m$, since $r<y$ and $\frac{(u-m+1) !}{u !} \leq 1$, we have

$$
\frac{r !}{\left(r-\frac{m-3}{2}\right) !} \frac{(u-m+1) !}{u !} \leq \frac{1}{6}(y \log y)^{\frac{m-1}{2}} \log y .
$$

This implies (4.8).

Recall that we are treating a composition $r_{1}+r_{2}+\cdots+r_{u}=2 r$ with $r_{1}=r_{2}=\cdots=r_{m}=1$. Let $N$ be the number of conjugacy classes of $G$, and partition the sum $\sum_{\rho \in L(X)}$ into $(N+w)^{u}$ sums, namely, given $\left(\mathcal{S}_{1}, \ldots, \mathcal{S}_{u}\right)$, where $\mathcal{S}_{i}$ is either $\mathcal{S}_{p_{i}, C}$ or $\mathcal{S}_{p_{i}, r_{j}}$, we consider the set of $\rho \in L(X)$ with the local conditions $\mathcal{S}_{i}$ for each $i$. Note that in each such partition, $a_{\rho}\left(p_{1}\right)^{r_{1}} \cdots a_{\rho}\left(p_{u}\right)^{r_{u}}$ remains a constant. 
Suppose $p_{1}$ is unramified, and fix the splitting types of $p_{2}, \cdots, p_{u}$, and let Frob $p_{1}$ runs through the conjugacy classes of $G$. Then by (2.2), the sum of such $N$ partitions is

$$
\sum_{C}\left(\frac{|C| a_{\rho}\left(p_{1}\right)}{|G|\left(1+f\left(p_{1}\right)\right)} A\left(\mathcal{S}_{2}, \ldots, \mathcal{S}_{u}\right) X+O\left(\left(p_{1} \cdots p_{u}\right)^{\kappa} X^{\delta}\right)\right),
$$

for a constant $A\left(\mathcal{S}_{2}, \ldots, \mathcal{S}_{u}\right)$. Let $\chi_{\rho}$ be the character of $\rho$. Then $a_{\rho}(p)=\chi_{\rho}(g)$, where $g=$ Frob $_{p}$. By orthogonality of characters, $\sum_{C}|C| a_{\rho}\left(p_{1}\right)=\sum_{g \in G} \chi_{\rho}(g)=0$. Hence the above sum is $O\left(\left(p_{1} \cdots p_{u}\right)^{\kappa} X^{\delta}\right)$. The contribution from these $N$ partitions to (4.5) is,

$$
\begin{aligned}
& \ll X^{\delta} \frac{(2 r) !}{r_{1} ! \cdots r_{u} !} \frac{1}{u !} \sum_{p_{1}, \ldots, p_{u}}^{(2)} p_{1}^{\kappa-1} \cdots p_{m}^{\kappa-1} p_{m+1}^{\kappa-r_{m+1}} \cdots p_{u}^{\kappa-r_{u}} \\
& \ll X^{\delta} \frac{(2 r) !}{r_{1} ! \cdots r_{u} !} \frac{1}{u !} \prod_{i=1}^{m}\left(\sum_{y<p_{i}<x} p_{i}^{\kappa-1}\right) \prod_{i=m+1}^{u}\left(\sum_{y<p_{i}<x} p_{i}^{k-r_{i}}\right) \\
& \ll 2^{u} X^{\delta} \frac{(2 r) !}{r_{1} ! \cdots r_{u} !} \frac{1}{u !} \frac{x^{u \kappa}}{(\log x)^{u}} \ll 2^{u} X^{\delta} \frac{(2 r) !}{r !} \frac{x^{u \kappa}}{(\log x)^{u}} y^{m+r-u}(\log y)^{u-r} \ll 2^{u} X^{\delta} \frac{(2 r) !}{r !}(\log X)^{u \kappa \beta+r} .
\end{aligned}
$$

Here we used Lemma 4.7 for the second last inequality.

Hence the contribution from the cases when $p_{j}$ is unramified for some $j \leq m$, is

$$
\ll(N+w)^{u} 2^{u} X^{\delta} \frac{(2 r) !}{r !}(\log X)^{u \kappa \beta+r} \ll(N+w)^{2 r} 2^{2 r} X^{\delta} \frac{(2 r) !}{r !}(\log X)^{2 r(\kappa \beta+1)} .
$$

If we choose $c_{2}$ sufficiently small, for example, taking $c_{2}=\frac{1-\delta}{20(\kappa \beta+1)}$,

$$
(N+w)^{2 r} 2^{2 r} X^{\delta} \frac{(2 r) !}{r !}(\log X)^{2 r(\kappa \beta+1)} \ll d^{2 r} X \frac{(2 r) !}{r !} \frac{2^{2 r}}{(y \log y)^{r}} .
$$

Hence we verified (4.5).

Now, we assume that $p_{1}, p_{2}, \cdots, p_{m}$ are all ramified. Then by (2.2), the number of elements in the set of $\rho \in L(X)$ with the local condition $\mathcal{S}_{p_{i}, r}$ for $i=1, \ldots, m$, is

$$
\prod_{i=1}^{m} \frac{f\left(p_{i}\right)}{1+f\left(p_{i}\right)} A\left(r_{2}\right) X+O\left(\left(p_{1} \cdots p_{m}\right)^{\kappa} X^{\delta}\right)
$$

Since $\frac{f(p)}{1+f(p)} \ll \frac{1}{p}$, by the trivial bound, the main term contributes to (4.5)

$$
\begin{aligned}
& X d^{2 r} \sum_{p_{1}, \ldots, p_{u}}^{(2)} \frac{1}{p_{1}^{2} \cdots p_{m}^{2} p_{m+1}^{r_{m+1}} \cdots p_{u}^{r_{u}}} \ll X d^{2 r} \prod_{i=1}^{m}\left(\sum_{y<p_{i}<x} p_{i}^{-2}\right) \prod_{i=m+1}^{u}\left(\sum_{y<p_{i}<x} p_{i}^{-r_{i}}\right) \\
& \ll X d^{2 r} 2^{2 r}(y \log y)^{-r} \frac{y^{u}}{y^{m+r}} \cdot \frac{(\log y)^{r}}{(\log y)^{u}} .
\end{aligned}
$$

By Lemma 4.7, (4.5) is verified.

The contribution of the error term $O\left(\left(p_{1} \cdots p_{m}\right)^{\kappa} X^{\delta}\right)$ is the same as when $p_{1}$ is unramified. 
Now take $y=c_{1} \log X$, and $r=c_{2} \frac{\log x}{\log \log X}$. Then from Proposition4.3, the number of $\rho \in L(X)$ such that $\left|\sum_{y<p<x} \frac{a_{\rho}(p)}{p}\right|>\frac{1}{(\log \log X)^{1 / 2}}$, is

$$
\ll X e^{-c^{\prime} \frac{\log X}{\log \log X} \log \log \log X},
$$

for some $c^{\prime}>0$. This proves Proposition 4.1

4.2. Infinite family of number fields with extreme residues. Let $C$ be a conjugacy class of $S_{d+1}$, and $\mathcal{S}=\left(S_{p, C}\right)_{p \leq y}$ be the set of local conditions such that for every prime $p \leq y$, Frob $_{p} \in C$. We denote $L(X, \mathcal{S})^{r_{2}}$ by $L(X, \mathcal{S})$. Conjecture 2.1 says that

$$
|L(X, \mathcal{S})|=A\left(r_{2}\right) X \prod_{p \leq y} \frac{\frac{|C|}{\left|S_{d+1}\right|}}{1+f(p)}+O\left(\left(\prod_{p \leq y} p\right)^{\gamma} X^{\delta}\right) .
$$

The main term is

$$
A\left(r_{2}\right) \frac{X}{\log y} \exp \left(-\log \frac{\left|S_{d+1}\right|}{|C|} \cdot \frac{\log X}{\log \log X}\right) .
$$

This is larger than (4.10). Also we may assume that almost all $L$-functions in $L(X, \mathcal{S})$ have the desired zero-free region of the form in Proposition 3.5. Hence, by Proposition 4.1, except $O\left(X e^{-c^{\prime} \frac{\log X}{\log \log X} \log \log \log X}\right)$ fields,

$$
L(1, \rho)=\prod_{\substack{p \leq y \\ F r o b p \in C}} \prod_{i=1}^{d}\left(1-\alpha_{i} p^{-1}\right)^{-1}\left(1+O\left(\frac{1}{\left(\log \log \left|D_{K}\right|^{\frac{1}{2}}\right.}\right)\right) .
$$

By taking $C=1$, we obtain an infinite family of number fields with the upper bound. On the other hand, by taking $C=(1, \cdots, d+1)$, we obtain an infinite family of number fields with the lower bound. This proves Theorem 1.3 .

In a similar way, for each $0 \leq i \leq d, d-i$ even, we can construct an infinite family of number fields with the residue

$$
\zeta(2)^{\frac{d-i}{2}} e^{\gamma i}\left(\log \log \left|D_{K}\right|\right)^{i}(1+o(1))
$$

In particular we obtain an infinite family of number fields with bounded residues by taking

$$
C=\left\{\begin{array}{ll}
(1,2)(3,4) \cdots(d-1, d), & \text { if } d \text { is even } \\
(1,2)(3,4) \cdots(d-4, d-3)(d-2, d-1, d), & \text { if } d \text { is odd }
\end{array} .\right.
$$


for which

$$
\operatorname{Res}_{s=1} \zeta_{K}(s)=L(1, \rho)=\left\{\begin{array}{ll}
\zeta(2)^{\frac{d}{2}}(1+o(1)), & \text { if } d \text { is even } \\
\zeta(2)^{\frac{d-3}{2}} \zeta(3)(1+o(1)), & \text { if } d \geq 3 \text { is odd. }
\end{array},\right.
$$

and it proves Theorem 1.4.

\section{REFERENCES}

[1] P.J. Cho and H.H. Kim, Probabilistic properties of number fields, J. Number Theory, 133 (2013), 4175-4187.

[2] — Central limit theorem for Artin L-functions, to appear in IJNT, arXiv:1506.07416.

[3] S. Chowla, Improvement of a theorem of Linnik and Walfisz, Proc. London Math. Soc. 50 (1949), 423-429.

[4] R.C. Daileda, Non-abelian number fields with very large class numbers, Acta Arith. 125 (2006), 215-255.

[5] A. Granville and K. Soundararajan, The Distribution of Values of $L\left(1, \chi_{d}\right)$, Geom. Funct. Anal. 13 (2003), no. 5, 992-1028.

[6] _ Large character sums, Journal of AMS 14 (2000), no. 2, 365-397.

[7] E. Kowalski and P. Michel, Zeros of families of automorphic L-functions close to 1, Pac. J. Math. 207 (2002), No. 2, 411-431.

[8] Y. Lamzouri, Extreme values of class numbers of real quadratic fields, IMRN, to appear.

[9] _ Large values of $L(1, \chi)$ for $k$-th order characters $\chi$ and applications to character sums, 18 pages, preprint.

[10] J.E. Littlewood, On the class number of corpus $P(\sqrt{-k})$, Proc. of the London Math. Soc. 27, no.1 (1928): 358-372.

[11] H.L. Montgomery and R.C. Vaughan, Extreme values of Dirichlet L-functions at 1, Number Theory in Progress, Vol. 2 (Zakopane-Kościelisko, 1997), 1039-1052, de Gruyter, Berlin, 1999.

[12] T. Taniguchi and F. Thorne, Secondary terms in counting functions for cubic fields, Duke Math. J. 162 (2013), $2451-2508$.

Department of Mathematical Sciences, Ulsan National Institute of Science and Technology, ULSAN, KOREA

E-mail address: petercho@unist.ac.kr

Department of Mathematics, University of Toronto, On M5S 2E4, CANADA, and Korea Institute For Advanced Study, Seoul, Korea

E-mail address: henrykim@math.toronto.edu 